\title{
Akademie Online startet mit neuem Schwerpunkt ins Programmjahr 2018
}

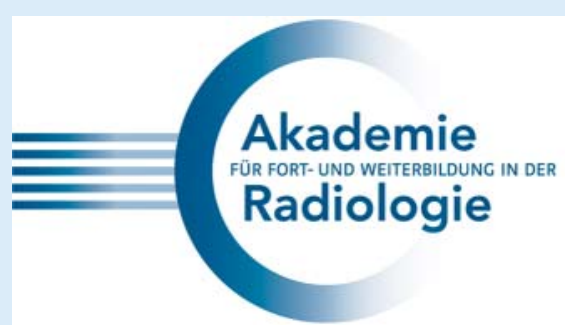

Für mittlerweile über 1500 Abonnenten bietet die Fortbildungsreihe „Akademie Online“ auch 2018 wieder ein abwechs- lungsreiches und praxisorientiertes Programm. Mit insgesamt 25 Kursen für ärztliches Personal und 12 Kursen für MTRA reicht die Themenspanne von den Basics der kardialen Diagnostik über entzündliche Darmerkrankungen bis zu odontogenen Tumoren und Nebennierenraumforderungen. MTRA erfahren etwas über die Grundlagen des neuen Strahlenschutzgesetzes im Bereich Teleradiologie, Stolperfallen im konventionellen Röntgen und typische Diagnosen an Schulter und Ellenbogen.
In nunmehr sieben Jahren „Akademie Online“ haben wir auch zahlreiche Themenvorschläge und -wünsche von unseren Teilnehmern erhalten, die uns geholfen haben, unser Programm kontinuierlich am Bedarf der Zuhörerschaft auszurichten. Ein Feedback haben wir dabei besonders oft gehört: „Mehr Neuro-Themen!“ Deswegen freuen wir uns sehr, ab dem Jahr 2018 die hohe Nachfrage nach neuroradiologischen Themen mit einem neuen Schwerpunktangebot zu beantworten: In Zusammenarbeit mit der 


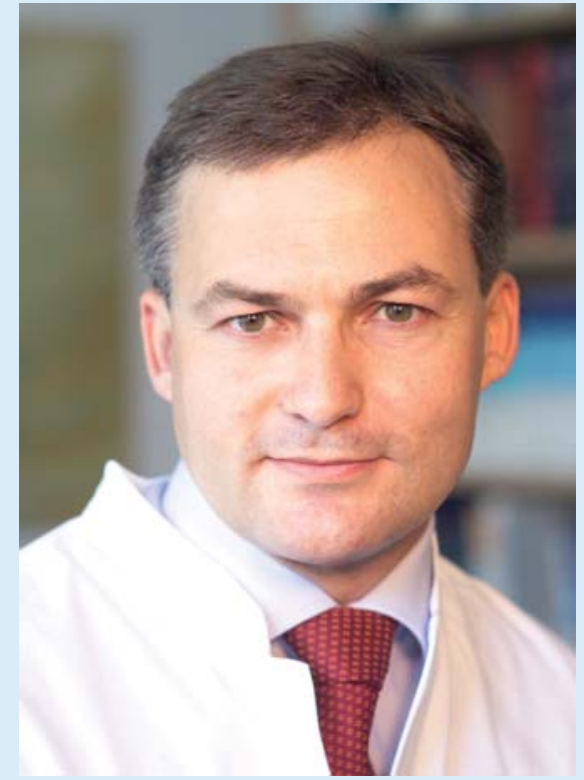

Prof. Dr. Arnd Dörfler

Deutschen Gesellschaft für Neuroradiologie (DGNR) bietet „Akademie Online“ sechs der insgesamt 25 ärztlichen Kurse pro Jahr unter dem Titel „Fokus Neurorad“ an.

„Die Leute wollen Köpfe sehen“, berichtet so auch Prof. Dr. Arnd Dörfler, Präsident der DGNR und Initiator der Programmreihe, zu den Hintergründen für die Schaffung des neuen Angebots. Er wird die Neuro-Kurse künftig gemeinsam mit Prof. Dr. Martin Mack moderieren. Lesen Sie mehr dazu im nebenstehenden Interview.

Den neuen Akademie-Online-Kursplan 2018 finden Sie ab sofort online unter www.drgakademie.de > Akademie Online. Hier können Sie sich auch für das Akademie-Online-Jahresabonnement 2018 anmelden: Mit nur einer Buchung nehmen Sie an allen 37 Online-Kursen teil. Zusätzlich erhalten Sie Zugang zum passwortge- schützten Stream-Bereich, in dem Sie verpasste Kurse für die Dauer von 14 bzw. 28 Tagen noch einmal als Aufzeichnung ansehen können. Als DRG- oder DGNR-Mitglied gibt es das Jahresabo bereits ab 80 Euro (AiW) bzw. 200 Euro (FA). VMTB-Mitglieder (MTRA) können sich bereits für 60 Euro pro Jahr anmelden. Noch günstiger wird es, wenn Sie das Jahresabo als Gruppe über den Arbeitgeber, d. h. Institut oder Praxis, buchen. Beim so genannten „Institutsabo“ sparen Sie als Gruppe noch einmal bis zu $50 \%$.

Erstmal unverbindlich reinschnuppern? Akademie Online können Sie jederzeit kostenfrei testen. Wählen Sie in unserem Kalender einfach eine der nächsten Veranstaltung aus und fordern Sie einen Zugangslink unter Angabe der Option „,kostenloser Testzugang“an.

Interview mit Prof. Dr. Arnd

Dörfler, Präsident der DGNR

Herr Professor Dörfler, was ist die Idee hinter der neuen Akademie-Online-Reihe?

Schon in den vergangenen Jahren spielte die Neuroradiologie eine herausragende Rolle im Programm der Online-Akademie der DRG. Seitens der Fachgesellschaft möchten wir diesen Trend aufgreifen und mit einer eigenen Programmreihe an insgesamt sechs Terminen im Jahr neuroradiologische Fortbildung anbieten - das betrifft rund 25 Prozent des ärztlichen Kursvolumens. Die Kurse werden gezielt beworben und finden selbstverständlich am gewohnten Fortbildungs-Dienstag statt.

\section{An welche Zielgruppe richtet sich das} Programm?

Der Bedarf an neuroradiologischer Fortbildung ist enorm. Steigende neuroradiologische Patientenzahlen, innovative Untersuchungsverfahren und nicht zuletzt fehlende Zeitkapazitäten für interne Fortbildungen lassen diesen Bedarf an guter, praxisorientierter Fortbildung wachsen - auch bei den Allgemeinradiologen. Die Leute wollen Köpfe sehen. Unser Programm in diesem Jahr verbindet relevante Themen mit zugstarken und namhaften Referenten. Und auch die Moderation, die bei der Online-Fortbildung eine zentrale Rolle spielt, wird in dieser Reihe neuroradiologisch besetzt sein. Wir sind zuversichtlich, hier innerhalb der Reihe der Akademie ein attraktives zusätzliches Angebot gesetzt zu haben sowohl für Neuroradiologen als auch für den Allgemeinradiologen.

\section{Gibt es Vergünstigungen?}

Alle Mitglieder unserer Fachgesellschaft erhalten eine Ermäßigung von $50 \%$ auf das Jahres-Abo Akademie Online. Für fachärztliche Gesellschaftsmitglieder bedeutet das 200 Euro pro Jahr, Weiterbildungsassistenten zahlen 80 Euro. Einen besonderen Anreiz gibt es für Neu-Mitglieder unserer Gesellschaft. Für diese übernehmen wir nämlich für ein Jahr die Kosten des Abos, wenn sie sich zwischen November 2017 und Januar 2018 für eine DGNR-Mitgliedschaft entscheiden.

Originalveröffentlichung des Interviews unter www.dgnr.org 